\title{
Supporting Information: Robust Giant Magnetoresistance in 2D Van der Waals Molecular Magnetic Tunnel Junctions
}

\author{
Dongzhe $\mathrm{Li},{ }^{1}$ Thomas Frauenheim, ${ }^{2}$ and Junjie $\mathrm{He}^{2,3, *}$ \\ ${ }^{1}$ Institute for Advanced Study, Chengdu University, Chengdu 610100, P. R. China \\ ${ }^{2}$ Bremen Center for Computational Materials Science, University of Bremen, Am Fallturm 1, \\ 2835, Bremen, Germany \\ ${ }^{3}$ Department of Physical and Macromolecular Chemistry \& Charles University Centre of \\ Advanced Materials, Faculty of Science, Charles University in Prague, Hlavova 8, Prague 2, \\ 128 43, Czech Republic
}

*E-mail: junjie.he.phy@gmail.com 


\section{Comparison between SIESTA and QUANTUM ESPRESSO}

The reliability of the basis sets parameters used in our SISESTA calculations has been checked by comparing the electronic structure with plane-wave QUANTUM ESPRESSO (QE) package. We plot in Fig. S1 the spin-resolved total DOS (black) and PDOS on Fe1 (blue) and Fe2 (red) atoms using S ISESTA (top) and QE (bottom). A very good quantitative agreement is found.
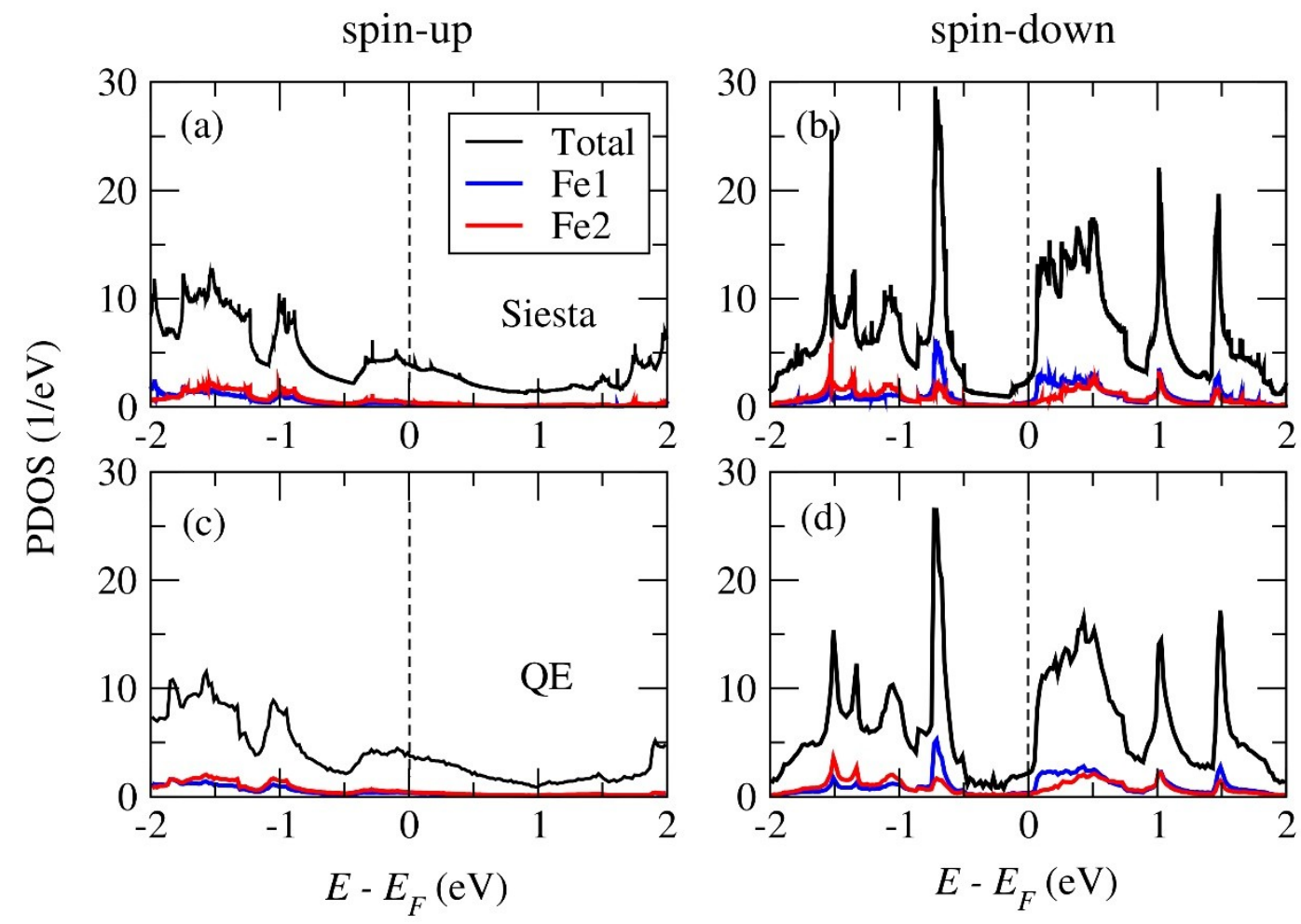

Figure S1: Comparison between SIESTA and QUANTUM E SPRESSO (QE) results. Calculated spindependent total DOS and PDOS on Fe1and Fe2 by SIESTA (a-b) and QE (c-d) for bulk $\mathrm{Fe}_{3} \mathrm{GeTe}_{2}$. A good quantitative agreement is found between both set of results, insuring the accuracy of the basis sets parameters used in SIESTA. 


\section{II. k-points convergence for transmission function}

We have carefully checked the $k$-point convergence for transmission function calculations. Using a 24 $\times 24$ in-plane $k$-point mesh in the $3 \times 3$ FGT supercell suffices to obtain converged results for the transmission, as is demonstrated by Fig. 2 .

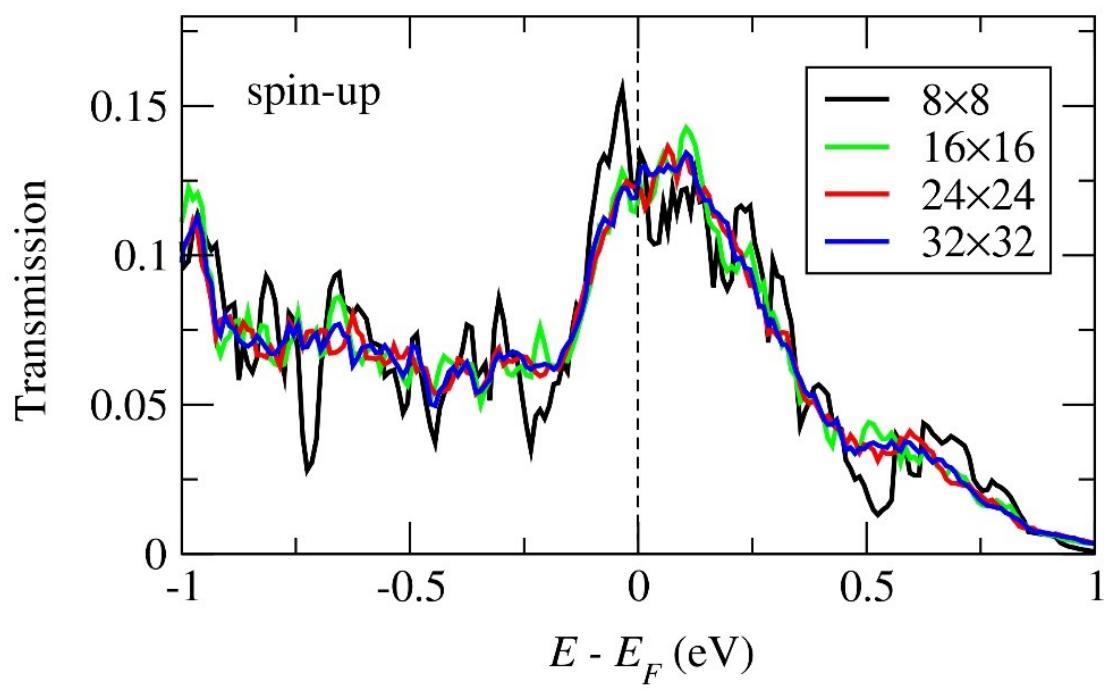

Figure S2: Spin-up transmissions for $\mathrm{Fe}_{3} \mathrm{GeTe}_{2} / \mathrm{BDT} / \mathrm{Fe}_{3} \mathrm{GeTe}_{2}$ at zero bias. The Fermi level is at zero energy. Curves are given for $8 \times 8,16 \times 16,24 \times 24$, and $32 \times 32 k$-point grid samplings.

\section{Molecular orbitals in the gas-phase}

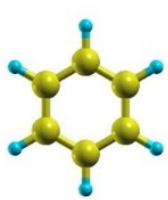

HOMO-1 (× 2)

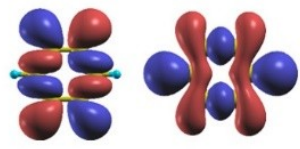

HOMO-1
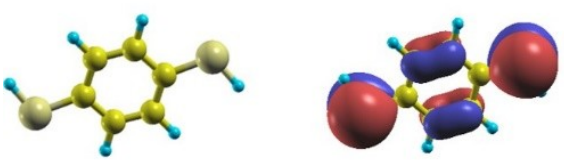

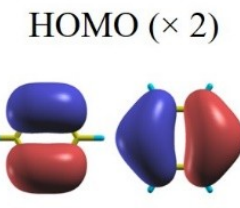

LUMO (× 2)

$\mathrm{LUMO}+1$
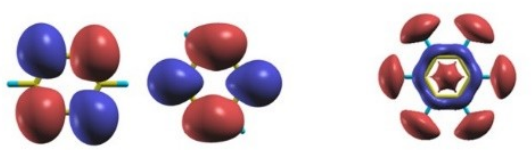

HOMO

LUMO

LUMO+1
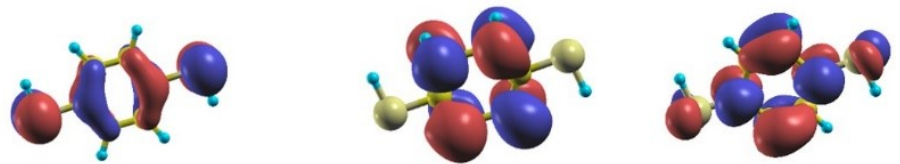

Figure S3: The spatial distributions of molecular orbitals in the gas-phase for benzene and BDT. All the orbitals are $\pi$-nature which are perpendicular to the molecular plane. Isosurfaces of positive and negative isovalues are shown in red and blue, respectively. 


\section{IV. $\mathrm{Fe}_{3} \mathrm{GeTe}_{2} / \mathrm{BDT}$ junctions}

\section{A. Absorption sites}
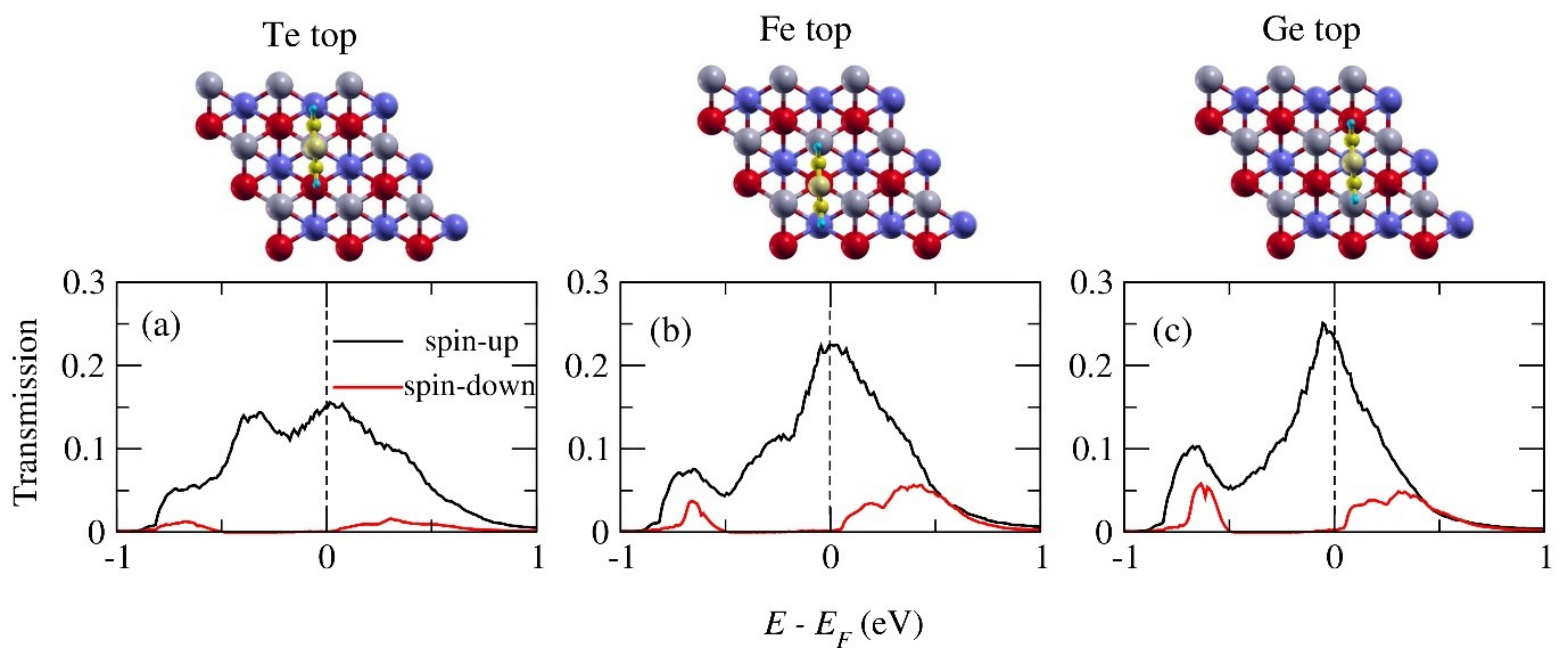

Figure S4: Transmission functions for $\mathrm{Fe}_{3} \mathrm{GeTe}_{2} / \mathrm{BDT}$ junctions with different connections at the interfaces where the S atom absorbed on different sites, namely Te top (a), Fe top (b), and Ge top (c). For the sake of simplicity, the top view of geometries without Au tip are presented. Black and red lines denote spin-up and -down channels, respectively.

\section{B. Interface defects}

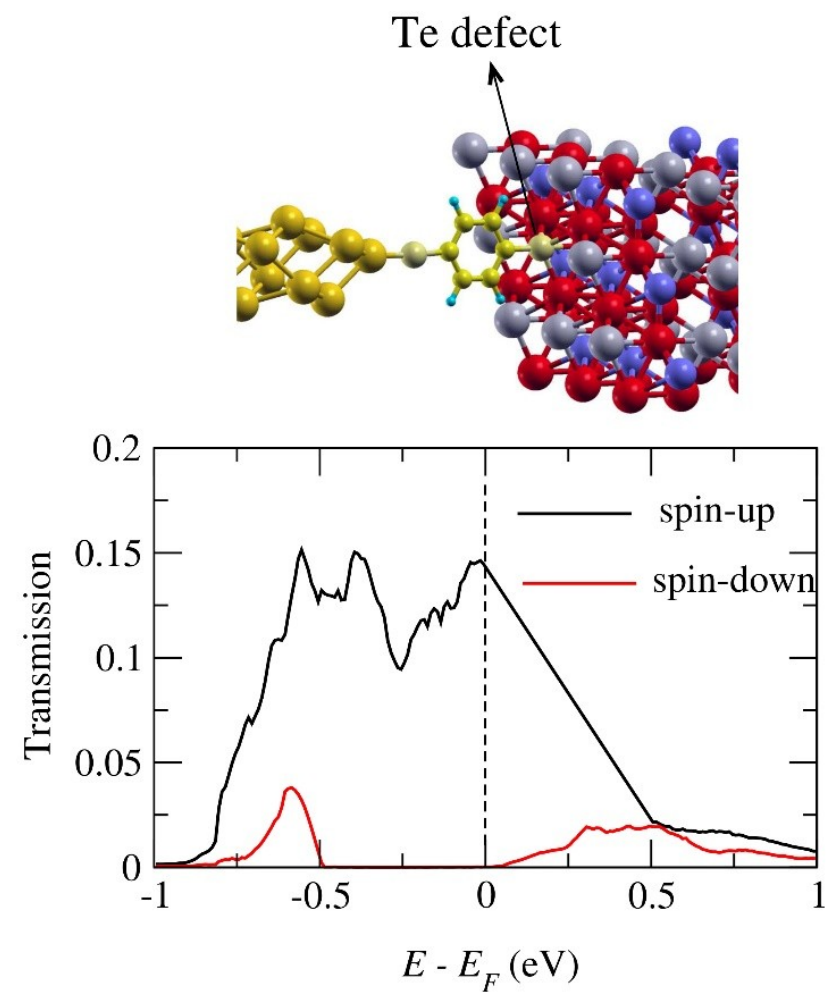

Figure S5: Transmission functions for $\mathrm{Fe}_{3} \mathrm{GeTe}_{2} / \mathrm{BDT}$ junctions with a single $\mathrm{Te}$ defect at the interfaces. Black and red lines denote spin-up and -down channels, respectively. 


\section{V. $\mathrm{Fe}_{3} \mathrm{GeTe}_{2} / \mathrm{BDT} / \mathrm{Fe}_{3} \mathrm{GeTe}_{2}$ molecular spin-valve}

\section{A. Stacking configuration}

(a) $\mathrm{ABA} / \mathrm{BDT} / \mathrm{ABA}$

(b) $\mathrm{ABAB} / \mathrm{BDT} / \mathrm{BAB}$
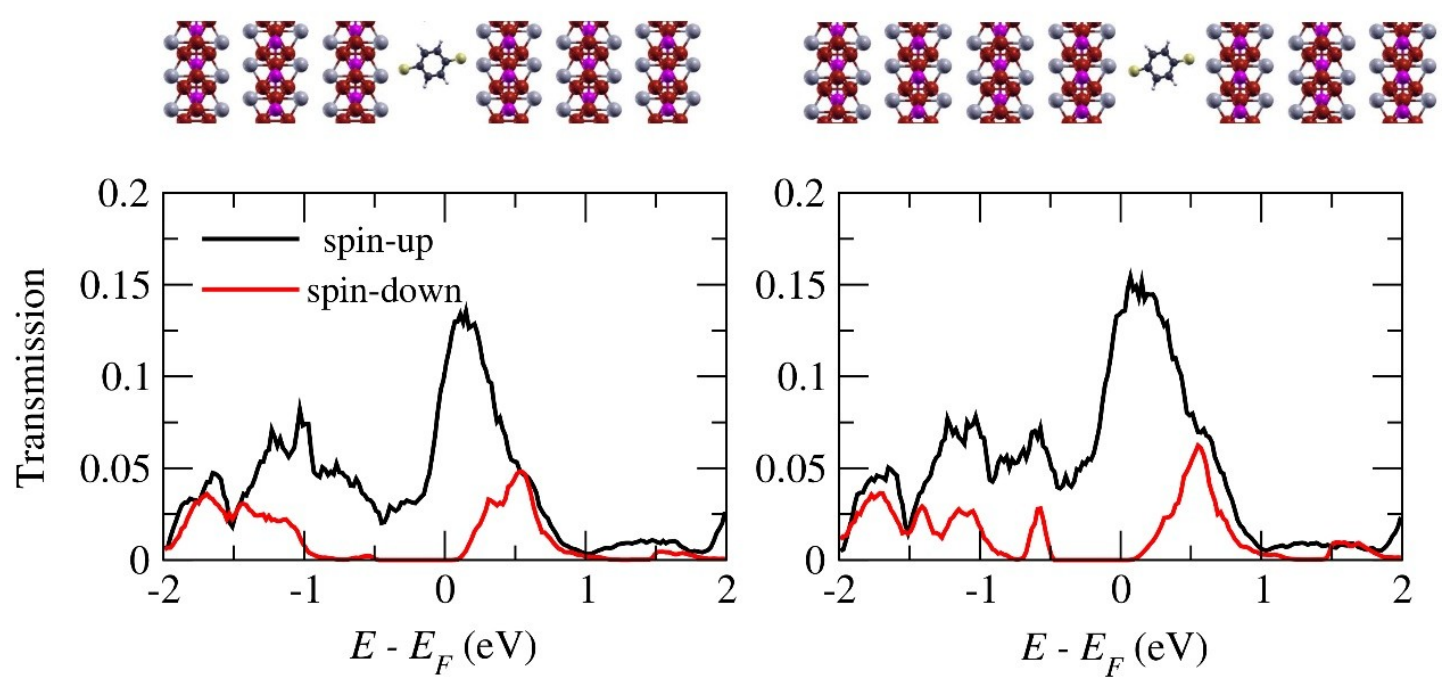

Figure S6: Spin-dependent transmission functions of $\mathrm{Fe}_{3} \mathrm{GeTe}_{2} / \mathrm{BDT} / \mathrm{Fe}_{3} \mathrm{GeTe}_{2}$ junctions for $\mathrm{P}$ configuration with different $\mathrm{Fe}_{3} \mathrm{GeTe}_{2}$ stacking configurations, namely ABA/BDT/ABA (a) and $\mathrm{ABAB} / \mathrm{BDT} / \mathrm{BAB}(\mathrm{b})$. The transmission functions in both configurations are similar to the one presented in the main text $(\mathrm{ABA} / \mathrm{BDT} / \mathrm{BAB})$, indicating the stacking independence of spin transport behavior.

\section{B. Out-of-equilibrium spin transport}

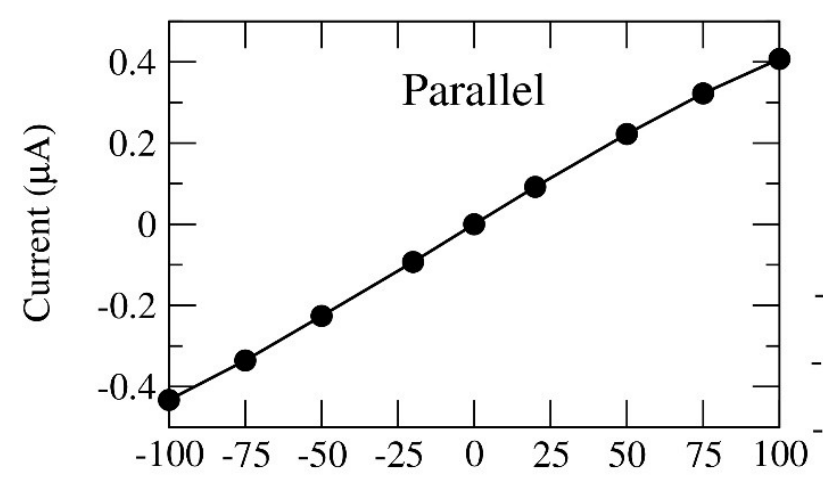

Bias voltage (meV)

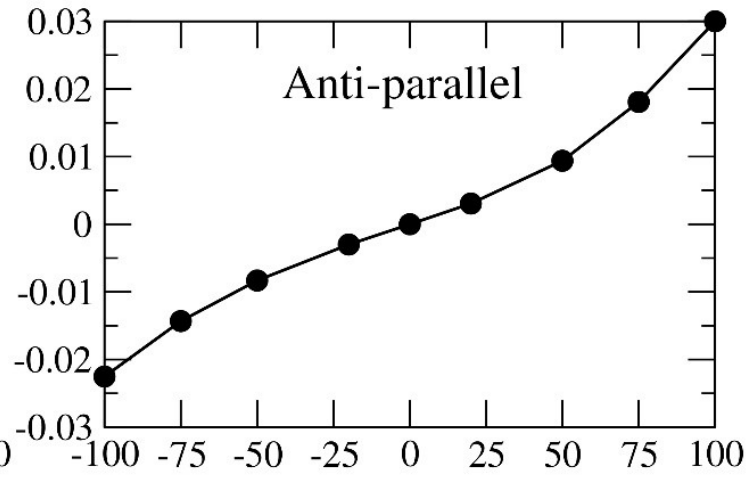

Bias voltage (meV)

Figure S7: The bias voltage dependence of current and for parallel (P) and anti-parallel (AP) spin configurations. $I-V$ curve for $\mathrm{P}$ configuration has linear dependence while a clear non-collinearity is seen in the case of AP configuration. 